Predictibilidad del mercado accionario colombiano

José Ignacio López-Gaviria 
Lecturas de Economía, 91 (julio-diciembre 2019), pp. 117-150

José Ignacio López-Gaviria

\section{Predictibilidad del mercado accionario colombiano}

Resumen: Este trabajo estudia los retornos históricos del mercado accionario colombiano y su predictibilidad en un horizonte de mediano y largo plazo, con el fin de establecer si la prima de riesgo es variante o constante en el tiempo y cuál es su relación con otras variables económicas. Para esto, se construye un indice de precios, retornos y dividendos para el periodo 1995-2017 con base en el universo de emisores del mercado de renta variable en Colombia. Se concluye que las fluctuaciones del ratio dividendo-precio del mercado accionario colombiano se explican principalmente por variaciones en los rendimientos futuros, lo que implica que el mercado está sujeto a ciclos y la prima de riesgo es variante en el tiempo. Adicionalmente, se encuentra que información sobre los créditos de vivienda, la tasa de cambio real y los retornos del indice S $\odot P$ 500 ayuda a aumentar el poder de predicción. Esto que sugiere que, para racionalizar la prima de riesgo de un mercado accionario como el colombiano, es útil pensar en modelos con mercados de crédito y en el contexto de una economía abierta.

Palabras clave: predictibilidad; prima de riesgo; rendimiento-dividendo; mercado accionario colombiano; retornos esperados.

Clasificación JEL: E44, G12, F23.

\section{Colombia's stock market predictability}

Abstract: This paper studies historical stock market returns in Colombia and their medium-and long-term predictability with the purpose of examining whether there is a constant or time-varying risk premium and its relationship with other economic variables. With this goal in mind, the paper presents a bistorical price index, returns and the aggregate dividend yield of Colombia's stock market for the 1995-2017 period, using information for the whole universe of issuers. Most of the variation in the dividend yield is explained by expected returns, which implies that the stock market has medium-and long-term cycles and the risk premium is time varying. The predictive power of the model increases if extended to include information on housing finance, the real exchange rate and returns of the S\&P 500 index, suggesting that credit frictions and small open economy considerations could play a role when modelling risk premium in Colombia's stock market.

Keywords: predictability; risk premium; dividend yield; Colombia's stock market; expected returns.

JEL Classification: E44, G12, F23.

\section{Prévisibilité de la bourse colombienne}

Résumé: Cet article étudie les rendements historiques du marché boursier colombien et sa prévisibilité à moyen et long terme, afin de déterminer si la prime de risque est variable ou constante dans le temps et quelle est sa relation avec d'autres variables économiques. . Pour cela, un indice de prix, de rendements et de dividendes est construit pour la période 1995-2017, considérant l'ensemble des émetteurs des titres à revenus variables sur marché des actions en Colombie. Il est conclu que les fluctuations du rapport prix/ dividende du marché boursier colombien s'expliquent principalement par les variations des rendements futurs, ce qui implique que le marché est soumis à des cycles et que la prime de risque est variable dans le temps. En outre, il a été constaté que les informations sur les prêts au logement, le taux de change réel et les rendements de l'indice S \& P500 contribuaient à accroître le pouvoir de prévision. Cela suggère que, pour rationaliser la prime de risque d'un marché boursier tel que le marché colombien, il est utile de réfléchir aux modèles utilisant les marchés du crédit dans le contexte d'une économie ouverte.

Mots clés: prévisibilité; prime de risque; dividende de rendement; marché boursier colombien; retours attendus.

Classification JEL: E44, G12, F23. 


\title{
Predictibilidad del mercado accionario colombiano
}

\author{
José Ignacio López-Gaviria $\mathbb{1}^{\mathrm{a}}$
}

\begin{abstract}
-Introducción. -I. Literatura relacionada. -II. Índice, retorno por dividendo y retornos totales del mercado accionario en Colombia. -III. Predictibilidad de retornos y prima de riesgo. -IV. Variables macroeconómicas y prima de riesgo. -Conclusiones. -Anexo. -Referencias.
\end{abstract}

doi: 10.17533/udea.le.n91a04

Primera versión recibida el 8 de junio de 2018; versión final aceptada el 14 de febrero de 2019

\section{Introducción}

El mercado accionario colombiano tiene un tamaño relativamente pequeño en relación al PIB del país y un bajo volumen de negociación, que no solo es muy inferior a otros mercados como el de renta fija, sino que ha estado estancado en los últimos años. El número de emisores es limitado, lo que implica que el mercado es relativamente poco diversificado. Así mismo,la incorporación de nuevos emisores en los últimos años no ha tenido la dinámica de otros mercados accionarios. Esto explica por qué la literatura se ha enfocado especialmente en temas de diseño institucional y de algunas de las ineficiencias que parecen regir en el mercado accionario colombiano.

A pesar de esto, el mercado accionario sigue siendo, por su naturaleza, el más propicio para estudiar la dinámica de riesgo agregado y la capacidad o disposición de los inversionistas para asumirlo. Este trabajo pretende llenar un vacío de la literatura estudiando los retornos históricos del mercado accionario y analizando la predictibilidad de dichos retornos en un horizonte de mediano y largo plazo (entre 2 y 5 años), esto con el fin de establecer si la

José Ignacio López-Gaviria: profesor, Universidad de los Andes, Colombia. Dirección postal: Carrera 1 No.18a-12. Dirección electrónica: ji.lopezg@uniandes.edu.co.

https://orcid.org/0000-0002-8210-123X 
prima de riesgo es variante o constante en el tiempo y cuál es su relación con otras variables económicas.

La primera contribución de este trabajo es la construcción de un índice de precios y dividendos desde 1995 hasta 2017, el cual permite tener una serie de retornos de más largo plazo a la que puede obtenerse con el índice COLCAP el índice de referencia calculado por la Bolsa de Valores de Colombia (BCV). Con base en la serie de retornos que aquí se presenta, es posible calcular algunos indicadores básicos del mercado accionario colombiano, que están ausentes en la literatura, como el retorno esperado incondicional (la media histórica) de los retornos del mercado accionario, en porcentaje y en unidades de desviación estándar, así como los retornos del mercado accionario en exceso de la tasa libre de riesgo. ${ }^{1}$

Para el período de análisis, el retorno anual promedio del índice de precios accionario que aquí se presenta es de 14,92\%, con una desviación estándar de 32,15\%, lo que implica un ratio de Sharpe (unidad de retorno por unidad de volatilidad) de 0,46 . El ratio dividendo-precio histórico del mercado accionario es de 4,15\%, lo que implica que el retorno total promedio del mercado accionario es de 19,07\% (que con una volatilidad de 31,66\% implica un ratio de Sharpe de 0,6). ${ }^{2}$

El exceso de retorno del mercado accionario, es decir el retorno total menos la tasa libre de riesgo, es de $8,49 \%$ para el período en estudio, lo que implica un ratio de Sharpe de 0,24, dada una desviación estándar de los excesos de retorno de $34,16 \%$.

Haciendo uso de la serie de retornos y el ratio dividendo-precio este trabajo discute si la prima de riesgo del mercado accionario es constante o cambia en el tiempo (un ejercicio conocido en la literatura como análisis de predictibilidad). ${ }^{3}$ Con base en los trabajos de Campbell y Schiller (1988a,

1 Los datos están disponibles en la página web del autor https://www.joseignaciolopez.com/cede.html

2 El factor del retorno total del mercado $\left(R_{t}\right)$ comprende el cambio en el precio $\left(P_{t+1} / P_{t}\right)$ y los dividendos agregados $\left(D_{t} / P_{t}\right): R_{t+1}=\frac{P_{t+1}+D_{t+1}}{P_{t}}$

3 Es importante aclarar que los modelos de predictibilidad tienen un nombre que puede ser engañoso. "Predictibilidad" en estos modelos no significa que se pretende predecir 
1088b), Cochrane $(2007,2011)$ se analiza la fuente de variación del ratio de dividendo-precios para el período en cuestión.

Rangvid, Schmeling y Schrimpt (2014) estudian la predictibilidad de mercado accionarios para diferentes países del mundo, entre ellos Colombia. Para analizar el caso de este país se basan en una serie de retornos con poca significancia estadística: datos trimestrales desde 1993 hasta 2009, para un total de 65 observaciones. La hipótesis de este trabajo es que el mercado accionario tiene ciclos de largo plazo en los cuales cambios en la prima de riesgo se manifiestan en fluctuaciones de mediano y largo plazo en el ratio dividendo-precio, y en el retorno esperado de los activos.

El ejercicio que aquí se presenta tiene un mayor poder estadístico que el de Rangvid et al. (2014), dado que utiliza datos con frecuencia mensual, en vez de trimestral, y para un período más largo de tiempo: 1995-2017, lo que implica un total de 265 observaciones. Los resultados de este análisis difieren de los de Rangvid et al. (2014), pues sugieren que los movimientos de mediano y largo plazo (un horizonte de 2 a 5 años) del ratio dividendo-precio son explicados, en su mayoría, por retornos futuros; sin embargo, son similares al los que Cochrane (2011) encuentra para Estados Unidos, y sugieren que los inversionistas tienen una prima de riesgo variante en el tiempo: un aumento del ratio dividendo-precio pronostica un aumento de los retornos futuros esperados.

¿Por qué un aumento del ratio dividendo precios pronostica un aumento de los retornos futuros? Como lo indica la fórmula de Campbell-Shiller un movimiento en el ratio de dividendo-precio tiene que predecir un cambio en la tasa de crecimiento de dividendos o un cambio en los retornos. La teoría clásica de finanzas predice que un aumento de este ratio debería pronosticar una caída en la tasa de crecimiento de dividendos futuros. Si los precios incorporan toda la información disponible, y la tasa de descuento de los agentes es constante, una mala noticia sobre la tasa de crecimiento de dividendos futuros del mercado debería generar una reducción en los

exactamente el comportamiento del mercado, sino que significa que en algún momento del tiempo el comportamiento esperado del mercado tiene una probabilidad diferente a otro momento; en otras palabras, quiere decir que el retorno esperado del mercado no es constante. 
precios del mercado accionario y, por tanto, traducirse en un aumento del ratio dividendo-precio.

No obstante, para el caso colombiano, este trabajo encuentra lo opuesto: los movimientos en el ratio dividendo-precio del mercado accionario se explican, en su mayoría, por cambios en retornos futuros y, por lo tanto, sugieren que la tasa de descuento (la prima de riesgo) de los inversionistas es cambiante en el tiempo. En este caso, por ejemplo, una caída en los precios accionarios, que conlleva a un aumento del ratio dividendo-precio, predice un aumento de los retornos futuros.

Dado que la prima de riesgo es variante en el tiempo, vale la pena preguntarse qué otras variables económicas son relevantes a la hora de predecir los retornos futuros esperados del mercado accionario. Para el caso de Estados Unidos, existe un número grande de estudios empíricos que investigan cómo la prima de riesgo depende de variables macroeconómicas como la inflación (Fama, 1981; Fama \& Schwert, 1977; Nelson, 1976), los agregados monetarios (Geske \& Roll, 1983; Pearce \& Roley, 1983), la producción (Cutler \& Poterb, 1989), las tasas de desempleo (Boyd, Hu \& Jagannathan, 2005), las tasas de interés (Ang \& Bekaert, 2007); y las primas de riesgo de bonos (Campbell, 1987). Otros estudios se han enfocado en estudiar las primas de riesgo usando un conjunto grande de variables (Chen, Ross \& Roll, 1986).

El presente trabajo contribuye a la literatura que se ha enfocado en el estudio del mercado accionario colombiano, pues al considerar un conjunto grande de variables macroeconómicas, amplía el análisis de predictibilidad. El espíritu de este ejercicio es similar al de Ludvigson y $\mathrm{Ng}$ (2009). No existe un criterio ex-ante para decidir cuáles son las variables relevantes para pronosticar la prima de riesgo, ya que como mencionamos anteriormente, la literatura ha propuesto un número importante de variables macroeconómicas. Ludvigson y Ng (2009), al analizar la prima de riesgo de los bonos del tesoro de los Estados Unidos, utilizan un total de 132 variables económicas. Para este trabajo se construye una base de datos de frecuencia mensual de 42 series económicas, unas domésticas y otras internacionales, con el fin de evaluar qué variables contienen mayor información sobre los retornos esperados del 
mercado accionario colombiano. La introducción de variables internacionales, a diferencia de los trabajos para Estados Unidos, se justifica en el hecho de que Colombia, al tener una economía pequeña y abierta, está sujeta a cambios en los flujos de capitales que están determinados por factores internacionales, como son el nivel de las tasas de interés internacional, la prima de riesgo de los inversionistas extranjeros, los retornos del mercado accionario en Estados Unidos, entre otros. La consideración de estas variables está sustentada en evidencia empírica que muestra que los retornos del mercado accionario en los Estados Unidos tienen poder de predicción en otros mercados (véase Rapach, Strauss \& Zhou, 2013).

Usando la batería de indicadores económicos, se presentan regresiones para horizontes de 2, 3, 4 y 5 años y se busca cuáles variables tienen el mayor poder de predicción para los retornos del mercado accionario. Para los horizontes de 2 y 3 años, se encuentra que la variable de dividendo-precio es menos relevante. Adicionalmente, tres variables económicas ayudan a explicar los retornos del mercado: el crecimiento del crédito de vivienda, la tasa de cambio real y los retornos del índice S\&P 500. Para la regresión de 5 años la variable de ratio-dividendo precio tiene el signo esperado (positivo) y es altamente significativa. Las dos variables que marginalmente aportan poder explicativo son la tasa de crecimiento de crédito de vivienda y la tasa de cambio real, ambas con un signo negativo. Para el caso del crecimiento del crédito de vivienda, este resultado sugiere que, cuando la economía experimenta una caída en el ritmo de crecimiento del crédito hipotecario, los retornos futuros del mercado accionario son más altos. De otro lado, una caída en la tasa de crecimiento de los créditos de vivienda está asociada a un aumento en el riesgo agregado, que afecta tanto a los hogares como a las entidades del sistema financiero. Este aumento de riesgo es consistente con un apetito por activos de menor riesgo y, por lo tanto, con un aumento de los retornos esperados del mercado accionario.

Los resultados de este trabajo están en línea con los de otros trabajos que, para otros países, encuentran que la prima de riesgo del mercado accionario varía en el tiempo. Así mismo, esta investigación encuentra que cuando al modelo básico de predictibilidad se le añaden otras variables económicas (relevantes para la dinámica de precios y retornos de las acciones como lo 
son variables relacionadas con el dinamismo del mercado de crédito, la tasa de cambio real y los retornos accionarios en Estados Unidos) el poder predictivo aumenta. Estos resultados sugieren que, a la hora de racionalizar la prima de riesgo de un mercado accionario como el colombiano, es útil pensar en modelos con mercados de crédito (fricciones financieras) y en el contexto de un economía abierta.

El resto de este documento tiene la siguiente estructura: la siguiente sección presenta una síntesis de la literatura relacionada. La sección II presenta el índice de precios y dividendos agregados para el período 1995-2017, así como las estadísticas más importantes relacionadas con los retornos agregados del mercado. La sección III presenta los resultados correspondientes al análisis de mediano y largo plazo, y la última sección presenta la conclusiones.

\section{Literatura relacionada}

Varios trabajos han hecho un recuento importante de la historia y la estructura del mercado accionario colombiano (véase por ejemplo VélezPareja, 2000; Restrepo, Zuluaga \& Guerra, 2002 y Ospina, 2007). Parte del análisis sobre el mercado de renta variable en Colombia se enfoca en los temas de eficiencia, en particular para un horizonte de corto plazo y con datos de frecuencia diarios. Los trabajos de Montenegro (2007), Alonso y García (2009), Perez-Villalobos y Mendoza-Gutierrez (2010), y Kristjanpoller y Muñoz (2012), son algunos ejemplos de trabajos que documentan desviaciones de condiciones de eficiencia en el mercado accionario, como lo es la presencia de anomalías como el efecto día. Arango, González y Posada (2002) discuten la relación de los retornos con la tasa de interés, mientras que Sierra, Duarte y Ortiz (2015), con base en la hipótesis de mercados adaptativos, analizan la predictibilidad de corto plazo mediante procesos auto-regresivos y la eficiencia del mercado usando la prueba de Lo y MacKinlay (1988). Por su parte, Bastidas (2008) analiza los excesos de retorno del mercado accionario colombiano usando variables económicas con base en una estimación de coeficientes estocásticos mediante un filtro de 
Kalman. Recientemente, Gómez-Sánchez y Astaiza-Gómez (2015) realizaron un estudio de la prima de riesgo observada y del ciclo económico.

Los efectos de la unificación de las bolsas regionales en la Bolsa de Valores de Colombia han sido estudiados por Ochoa y Avendaño (2005). De otro lado, Yepes-Ríos, González-Tapia y González-Perez (2015) analizan la integración de los mercados de algunas economías latinoamericanas, entre ellos el colombiano, en el Mercado Integrado Latinoamericano (MILA) y sus efectos en la estructura del mercado.

La literatura sobre predictibilidad de largo plazo de los retornos accionarios es amplia, pero son pocos los estudios para países emergentes. La predictibilidad de retornos en el mercado accionario de Estados Unidos ha sido estudiada, por ejemplo, por Campbell y Schiller (1988a,1988b), Campbell y Ammer (1993), Goyal y Welch (2003), Lettau y Ludvignson (2005), Ang y Bekaert (2007), Cochrane (2007, 2011), Larrain y Yogo (2008), entre otros. En el contexto internacional, Harvey (1991), Solnik (1993) y Hjalmarsson (2010), entre otros, han estudiado la predictibilidad de diferentes mercados accionarios.

Finalmente, Rangvid et al. (2014) discute la predictibilidad de 50 mercados accionarios, entre ellos el colombiano, usando datos trimestrales para una muestra no balanceada entre 1973 y 2009. Estos autores encuentran que para Colombia los movimientos del ratio dividendo-precio se explican en partes iguales por cambios en retornos futuros y la tasa de crecimiento de dividendos futuros. Un ejercicio con datos mensuales, como el que aquí se presenta, donde las estimaciones tienen un mayor poder estadístico, sugiere que la mayoría de los movimientos del ratio dividendo-precio son explicados por cambios en retornos esperados, como en el caso del mercado accionario de Estados Unidos.

\section{II. Índice, retorno por dividendo y retornos totales del mercado accionario en Colombia}

Con el fin de tener una serie de tiempo histórica de los retornos del mercado accionario, se construye un índice de precios agregados y una serie de 
dividendos agregados para todo el universo de emisores en Colombia, desde marzo de 1995 hasta julio de 2017. Los datos anteriores a 2001, cuando se creó la Bolsa de Valores de Colombia que unificó la Bolsa de Bogotá, Medellín y Occidente, pertenecen a los registros de precios y volumen transado de cada una de las bolsas regionales.

El universo total de emisores con información es de 359, pero la mayoría de títulos no tiene información suficiente de precios y cantidades, lo que restringe de manera significativa el número de acciones consideradas. La Figura 1 muestra el número de acciones en cada momento del tiempo que cumplen con el requisito de tener información disponible para calcular cambios porcentuales en el precio, volúmenes de operación y capitalización bursátil. Como puede verse, el número es creciente en el tiempo, pero se estabiliza alrededor de 30 emisores, bajo el criterio de que exista información sobre la capitalización bursátil. Este universo de emisores es mayor al número de empresas incluidas en el índice COLCAP que está compuesto, en promedio, por las 20 acciones más líquidas del mercado. ${ }^{4}$ A diferencia del COLCAP y de otros índices accionarios internacionales, el índice agregado que aquí se presenta no cuenta con un criterio definido del número de emisores en la canasta de composición del índice. El número de emisores, como se mencionó anteriormente, cambia en el tiempo, y depende de la disposición de información: sobre precios y cantidades. El índice en cuestión tampoco restringe el universo de emisores por criterios de liquidez (bursatilidad), pero existe una alta correlación entre las acciones más líquidas y las que participan en el índice. Otra diferencia importante con el índice COLCAP es que el índice que aquí se construye no tiene topes específicos al peso de cada acción en la canasta. En últimas, lo que busca el índice propuesto es medir el retorno del inversionista representativo de la economía, es decir un inversionista que es dueño de todo el mercado accionario.

Con base en la información de precios, volúmenes, capitalización y dividendos es posible crear un índice de precios y dividendos agregado del mercado. Dado que no existe un criterio único de cómo agregar las

4 La metodología para el cálculo del COLCAP se puede encontrar en: http://www.bvc.com.co/pps/tibco/portalbvc/Home/Mercados/ descripciongeneral $/$ indicesbursatiles?action $=$ dummy 
acciones, el índice aquí propuesto se construye con base en ponderadores de capitalización bursátil y volumen transado. El índice se re-balancea trimestralmente, como el S\&P500 y otros índices internacionales. También se considera un índice alternativo, con cambio de ponderados anuales, que presenta un comportamiento similar.

Figura 1. Compañias activas en el mercado accionario colombiano

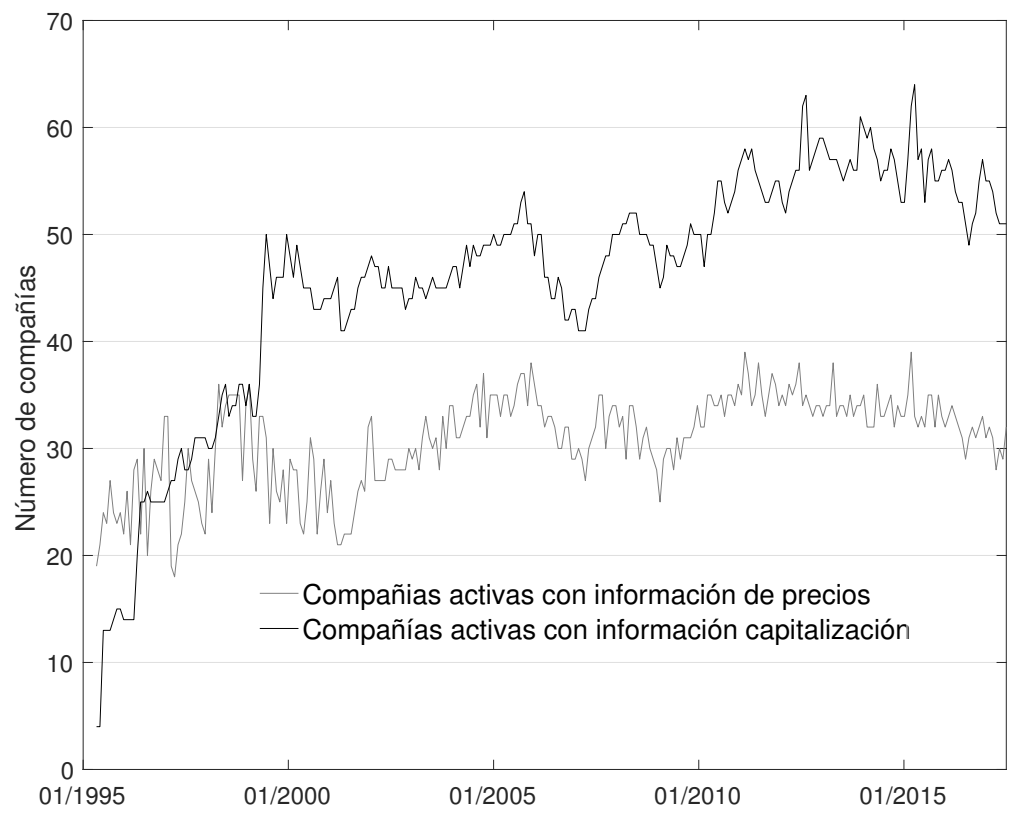

Fuente: elaboración propia con base en datos de la Bolsa de Valores de Colombia.

La Figura 2 muestra el coeficiente de Gini de los ponderados sobre el universo de emisores. Como puede verse, en el panel A, los ponderados tienen una alta concentración en pocas acciones, en particular porque muchos emisores no hacen parte del índice así que, incluso en el caso de igual peso para las acciones con información disponible, los ponderados muestran un algo nivel de concentración. En el panel B se presenta el coeficiente de Gini, normalizado para el caso de pesos igualitarios, que controla por el hecho de que no hay información para un número grande de emisores. 
Figura 2. Compañias activas en el mercado accionario colombiano

Panel $A$

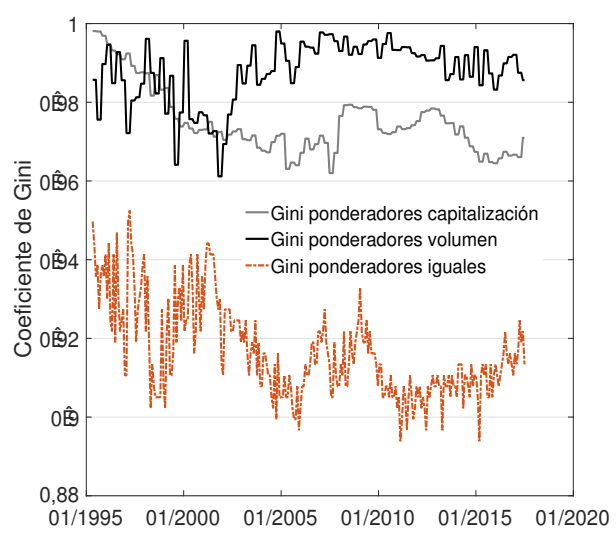

Panel B

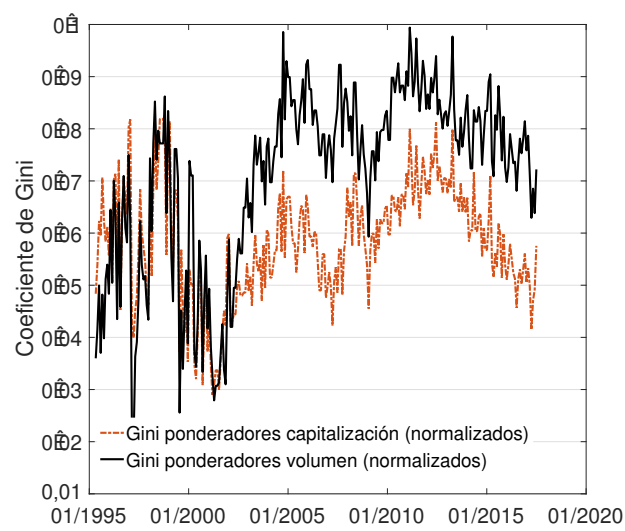

Fuente: elaboración propia con base en datos de la Bolsa de Valores de Colombia.

Como se evidencia, la concentración de los ponderadores es alta al inicio de la muestra, pero con la incorporación de más emisores se reduce hacia comienzos de la década pasada, para aumentar de nuevo debido a la incorporación de Ecopetrol.

La Figura 3 muestra el retorno anualizado con frecuencia mensual del índice del mercado accionario colombiano bajo los dos tipos de ponderadores. En términos generales, el índice muestra un comportamiento similar bajo los dos criterios de agregación. Los retornos positivos de los primeros años son sucedidos, durante la crisis económica de finales de los 90, de retornos negativos. El índice se recupera a comienzos de la década pasada y obtiene retornos significativamente altos a mediados de los 2000. Después de varios años de retornos anuales altos, el mercado accionario se corrige y vuelve a terreno negativo con la desaceleración económica de 20082009. Desde entonces el comportamiento del índice accionario exhibe una senda menos volátil, pero con algunos años de retornos negativos seguidos por rendimientos positivos hacia el final del período. 
Figura 3. Retorno anual del indice accionario bajo dos ponderadores

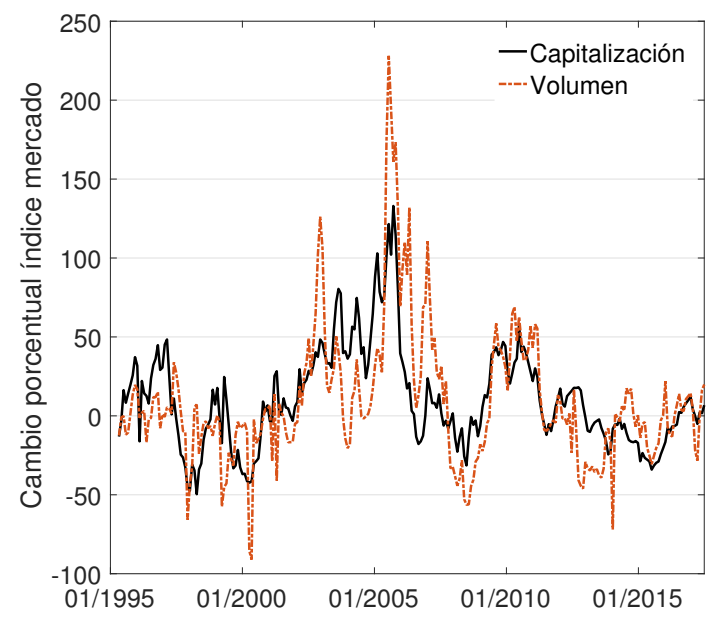

Fuente: elaboración propia.

Dado que el índice construido con base en ponderados de capitalización bursátil es menos volátil que en el caso de los ponderados por volumen, se usa el primero como el índice de referencia. El panel A de la Figura 4 grafica el índice del mercado accionario, normalizado a 100 para marzo de 1995.

¿Cómo se compara el desempeño de este índice accionario histórico con el del COLCAP? El panel B de la Figura 4 compara los retornos de los dos indicadores. Como puede verse, tanto el índice que aquí se presenta como el COLCAP tienen un comportamiento similar en términos de retornos anuales; sin embargo, el primero tiene la virtud de tener una serie histórica más larga y un número mayor de acciones, pues el COLCAP solo está disponible desde 2002 y está compuesto por 20 emisores. El criterio de selección de emisores en el COLCAP incluye características de liquidez y volumen de las acciones, y fija topes al máximo ponderador de cada acción.

A pesar del comportamiento similar, el COLCAP muestra unos retornos más altos que el índice del mercado que aquí se presenta: mientras el promedio de los retornos anuales con frecuencia mensual del COLCAP, desde agosto de 2002 hasta julio de 2017, es de 20,8 \%, para el índice del mercado accionario que aquí se construye es ligeramente inferior: 18,6\%. Esta diferencia se 
explica por la selección de los emisores y los ponderadores. En particular, el índice de referencia de este trabajo tiene más acciones y puede tener emisores con mayor peso, dado que los ponderadores no están limitados como en el caso del COLCAP. Esto puede generar que algunas empresas, relativamente grandes y con retornos más bajos, estén sobre-representadas en el índice con respecto al COLCAP, y por tanto que los retornos del COLCAP sean mayores.

Figura 4. Indice accionario y retorno del indice COLCAP

Panel A

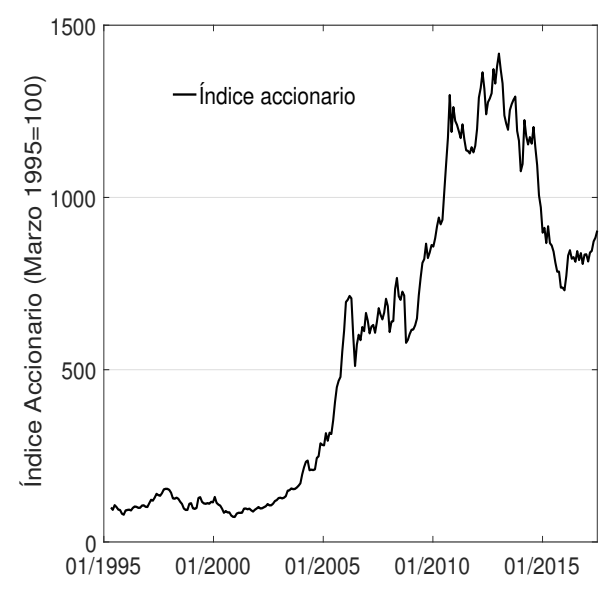

Panel B

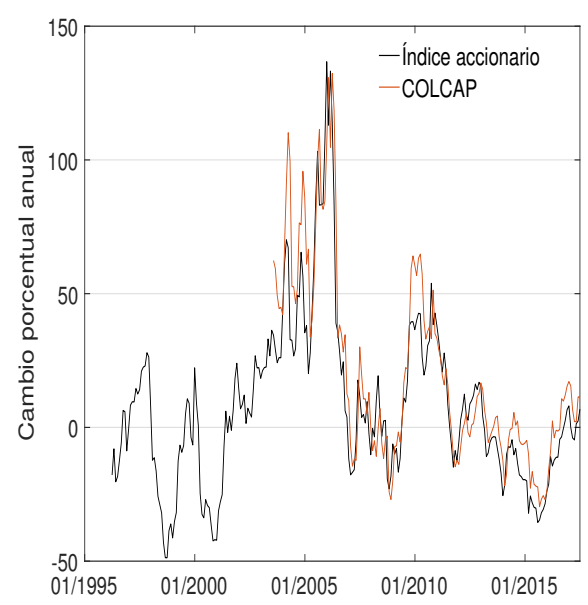

Fuente: elaboración propia.

Usando información sobre los dividendos de los emisores considerados en el índice, es posible construir, además, una serie agregada de dividendos con la cual podemos calcular el rendimiento por dividendo (ratio dividendoprecio) de todo el mercado. Cuando sumamos el retorno asociados con los dividendos, el retorno total del índice accionario es de $22,42 \%$.

La Figura 5 muestra el comportamiento histórico del rendimiento por dividendo para el mercado accionario colombiano. El rendimiento por dividendo de un acción indica cuánto recibe un inversionista por una unidad monetaria invertida. En el caso del rendimiento por dividendo de todo el 
mercado, este indicador muestra el flujo de ganancias de todo el mercado en forma de dividendos en relación al precio de todo el mercado accionario.

Figura 5. Rentabilidad por dividendo del mercado accionario colombiano

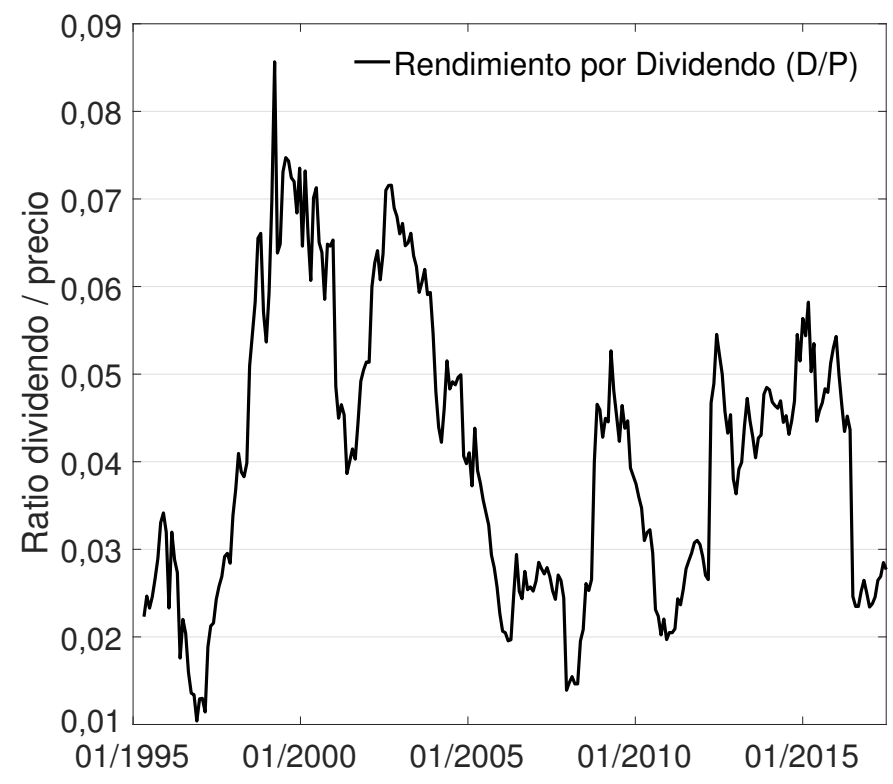

Fuente: elaboración propia.

Por medio del uso de los datos de dividendos es posible calcular el promedio histórico del retorno total del mercado accionario desde 1995 hasta 2017. La Tabla 1 resume las estadísticas históricas más importantes sobre retornos y excesos de retorno de las acciones en Colombia. Para todo el período de análisis el promedio del retorno anual del índice accionario fue de $14,92 \%$, con una desviación estándar de 32,15\%. Esto implica un ratio de Sharpe (unidad de retorno por unidad de volatilidad) de 0,46 , que coincide con los valores de otros índices internacionales.

Cuando sumamos el retorno por dividendos, el retorno total promedio de acciones para el período es de 19,07\% con volatilidad de 31,66\%. Para analizar el exceso de retorno tomamos la DTF a 90 días como la tasa libre de riesgo, la cual se decide usar, en parte, por su disponibilidad histórica. 
López-Gaviria: Predictibilidad del mercado accionario colombiano

El promedio de la DTF para el período $1995-2017$ es de $10,57 \%$, con una volatilidad de $8,42 \% .^{5}$

Tabla 1. Estadisticas del mercado accionario colombiano

\begin{tabular}{lccc}
\hline & \multicolumn{3}{c}{ Variables Nominales } \\
\hline Índice accionario & $\begin{array}{c}\text { Retorno total } \\
\text { Acciones }\end{array}$ & $\begin{array}{c}\text { Tasas de } \\
\text { interés }\end{array}$ \\
\hline Retorno anual \% (1) & 14,92 & 19,07 & 10,57 \\
Desviación estándar (2) & 32,15 & 31,66 & 8,42 \\
Ratio de Sharpe (1/2) & 0,46 & 0,60 & 1,25 \\
\hline & Índice accionario & Retorno total & Excesos de \\
& & Acciones & retorno \\
\hline Retorno anual \% (1) & 7,47 & 11,61 & 8,49 \\
Desviación estándar (2) & 31,21 & 30,74 & 34,16 \\
Ratio de Sharpe (1/2) & 0,24 & 0,37 & 0,24 \\
\hline
\end{tabular}

Nota: estadísticas para el período marzo 1995-julio 2017.

Fuente: elaboración propia.

El exceso de retorno histórico para el mercado accionario es de 8,49\% con una desviación estándar de 34,16\%, lo que implica un ratio de Sharpe de 0,24 , el cual resulta inferior al exceso de retorno histórico para los Estados Unidos, que es aproximadamente de 0,41 .

5 Buena parte de la literatura usa la tasa de bonos gubernamentales, como los tesoros americanos para el caso de Estados Unidos, como la tasa libre de riesgo. Es posible usar la tasa de los títulos de deuda pública doméstica emitidos por el gobierno, los TES, como la tasa libre de riesgo, pero en ese caso la muestra se reduce de manera importante, dado que el mercado de deuda pública, si bien comienza desde 2001 cuando el gobierno decide sustituir deuda externa por local, solo se consolida hasta mediados de los 2000. 


\section{Predictibilidad de retornos y prima de riesgo}

En esta sección se utiliza la serie de tiempo de los retornos del mercado, los dividendos y el rendimiento por dividendo del mercado accionario para evaluar si el rendimiento por dividendo ayuda a pronosticar los retornos o la tasa de crecimiento de los dividendos futuros.

Este ejercicio parte de la fórmula de Campbell-Shiller que relaciona el rendimiento por dividendo con los rendimientos, el crecimiento de dividendos y el rendimiento por dividendos futuros. Partiendo de la definición de retorno, podemos escribir los retornos del mercado accionario como:

$$
R_{t+1}=\frac{P_{t+1}+D_{t+1}}{P_{t}}=\frac{\frac{P_{t+1}}{D_{t+1}}+1}{\frac{P_{t}}{D_{t}}} \frac{D_{t+1}}{D_{t}},
$$

donde $P_{t}$ es el índice accionario y $D_{t}$ son los dividendos agregados del mercado. Tomando logaritmos a ambos lados tenemos que los retornos pueden definirse de la siguiente forma:

$$
r_{t+1}=\log \left(\frac{P_{t+1}}{D_{t+1}}+1\right)+\Delta d_{t+1}-\left(p_{t}-d_{t}\right),
$$

donde las variables en letras minúsculas están expresadas en logaritmos: $r_{t+1}=\log \left(R_{t+1}\right)$. Haciendo una aproximación de Taylor de primer orden alrededor del valor promedio del rendimiento por dividendo para la historia de la serie, es posible re-escribir el primer término del lado derecho de la ecuación anterior de la siguiente forma:

$$
\begin{aligned}
\log \left(\frac{P_{t+1}}{D_{t+1}}+1\right) \approx \log (P / D & +1) \\
& +\frac{P / D}{P / D+1}\left(p_{t+1}-d_{t+1}-(p-d)\right),
\end{aligned}
$$

donde $P / D=e^{p-d}$ es el valor promedio histórico del inverso del rendimiento por dividendo. Si definimos $\rho=\frac{P / D}{P / D+1}$ tenemos que $\rho \approx 0,99$, que es consistente con un rendimiento por dividendo mensual de $0,34 \%$. A 
partir de la aproximación de primer orden, el logaritmo de los retornos se define como:

$$
r_{t+1}=\kappa+\rho\left(p_{t+1}-d_{t+1}\right)+\triangle d_{t+1}-\left(p_{t}-d_{t}\right),
$$

donde $\kappa$ es una constante igual a $\log (P / D+1)$. Tomando el valor esperado, y expresando las variables como desviaciones de su promedio histórico, llegamos a la siguiente expresión:

$$
\hat{r}_{t+1}=\rho\left(\hat{p}_{t+1}-\hat{d}_{t+1}\right)+\triangle \hat{d}_{t+1}-\left(\hat{p}_{t}-\hat{d}_{t}\right),
$$

donde todas las variables están definidas como desviaciones de su media: $\hat{r}_{t+1}=r_{t+1}-E\left[r_{t+1}\right]$. De la ecuación anterior (omitiendo los gorros), podemos escribir el (log) rendimiento por dividendo como la resta de los retornos y el crecimiento de los dividendos más el valor (descontando) del rendimiento por dividendo del siguiente período:

$$
d_{t}-p_{t}=r_{t+1}-\triangle d_{t+1}+\rho\left(d_{t+1}-p_{t+1}\right) .
$$

Iterando hacia adelante, llegamos a la fórmula de Campbell-Shiller:

$$
d_{t}-p_{t}=\sum_{j=1}^{k} \rho^{j-1}\left(r_{t+j}-\triangle d_{t+j}\right)+\rho^{k}\left(d_{t+k}-p_{t+k}\right) \text {. }
$$

Esta ecuación nos dice que desviaciones del ratio dividendo-precio, frente a su promedio, deben ser iguales a las desviaciones de los retornos y la tasa de crecimiento de dividendos futuros, y un término que captura el ratio dividendo-precio en un horizonte lejano (este término es conocido en inglés como rational bubble).

Podemos regresar la tasa de crecimiento de dividendos, los retornos futuros y el rendimiento por dividendo utilizando como variable explicativa el ratio dividendo-precio:

$$
\sum_{j=1}^{k} \rho^{j-1} \triangle d_{t+j}=\triangle d_{t}^{L P}=\beta^{d g}\left(d_{t}-p_{t}\right)+\varepsilon_{d t},
$$




$$
\begin{gathered}
\sum_{j=1}^{k} \rho^{j-1} r_{t+j}=r_{t}^{L P}=\beta^{r}\left(d_{t}-p_{t}\right)+\varepsilon_{r t}, \\
\rho^{k}\left(d_{t+k}-p_{t+k}\right)=d p_{t}^{L P}=\beta^{d p}\left(d_{t}-p_{t}\right)+\varepsilon_{k t},
\end{gathered}
$$

donde los coeficientes tienen que cumplir la siguiente igualdad:

$$
\beta^{r}-\beta^{d g}+\beta^{d p} \approx 1
$$

El propósito de esta regresión no es establecer la causalidad entre estas variables, que está dada por una identidad, sino descomponer los movimientos del rendimiento por dividendo en las tres variables. El ratio dividendo-precio puede moverse si y solo si hay noticias sobre dividendos actuales, crecimiento de dividendos futuros o rentabilidad futura del mercado. Esta relación entre la variabilidad del rendimiento por dividendo, y rendimientos y dividendos futuros, se deduce de la definición de cada uno de los coeficientes de las tres anteriores regresiones:

$$
\begin{aligned}
\operatorname{var}\left(d_{t}-p_{t}\right)=\operatorname{cov}\left(r_{t}^{L P}, d_{t}-p_{t}\right)-\operatorname{cov}( & \left.\Delta d_{t}^{L P}, d_{t}-p_{t}\right) \\
& +\operatorname{cov}\left(d p_{t}^{L P}, d_{t}-p_{t}\right) .
\end{aligned}
$$

Por lo tanto, la varianza del rendimiento por dividendo precio se descompone en los tres componentes de covarianza.

\section{A. Retornos anuales con frecuencia anual}

La mayor parte de la literatura para el mercado accionario de los Estados Unidos ha hecho estimaciones con base en rendimientos acumulados, tasa de crecimiento de dividendos y el ratio dividendo-precio de series anuales. La disponibilidad histórica de estas series ha hecho posible realizar regresiones con datos anuales y horizontes de tiempo largo; sin embargo para el caso colombiano la escasa disponibilidad de datos impone restricciones a este ejercicio. Para solucionar este inconveniente se realizan dos tipos de 
regresiones diferentes: una con series anuales, usando la metodología de vectores auto-regresivos, y una con datos mensuales, la cual permite tener un número mayor de observaciones.

Los datos anuales de la serie histórica de este trabajo tienen 21 observaciones. Para hablar de predictibilidad de largo plazo se construye un vector autoregresivo que incluye el retorno anual del mercado, la tasa anual de crecimiento de los dividendos y el ratio anual de dividendo-precio:

$$
\left[\begin{array}{c}
r_{t+1}^{m} \\
\Delta d_{t+1} \\
d_{t+1}-p_{t+1}
\end{array}\right]=\Lambda\left[\begin{array}{c}
r_{t}^{m} \\
\Delta d_{t} \\
d_{t}-p_{t}
\end{array}\right]+\left[\begin{array}{c}
\varepsilon_{t+1}^{r} \\
\varepsilon_{t+1}^{d g} \\
\varepsilon_{t+1}^{d p}
\end{array}\right]
$$

Con base en la fórmula de Campbell-Shiller, la matriz $\Lambda$ es de la siguiente forma:

$$
\Lambda=\left[\begin{array}{ccc}
0 & 0 & b^{r} \\
0 & 0 & b^{d g} \\
0 & 0 & b^{d p}
\end{array}\right]
$$

Esta forma de definir el proceso estocástico de retornos, el crecimiento de dividendos y el ratio dividendo-precio de forma conjunta, permite encontrar los coeficientes de largo plazo iterando hacia adelante:

$$
\begin{gathered}
r_{t+1}^{m}=b^{r}\left(d_{t}-p_{t}\right)+\varepsilon_{t+1}^{r}, \\
r_{t+2}^{m}=b^{r} b^{d p}\left(d_{t}-p_{t}\right)+\varepsilon_{t+1}^{r}+\varepsilon_{t+2}^{r}, \\
r_{t+3}^{m}=b^{r}\left(b^{d p}\right)^{2}\left(d_{t}-p_{t}\right)+\varepsilon_{t+1}^{r}+\varepsilon_{t+2}^{r}+\varepsilon_{t+3}^{r},
\end{gathered}
$$

continuando la iteración hacia adelante tenemos que:

$$
\sum_{i=1}^{\infty} \rho^{i-1} r_{t+j}^{m}=b^{r}\left(1+\rho b^{d p}+\left(\rho b^{d p}\right)^{2}+\ldots\right)\left(d_{t}-p_{t}\right)+\sum_{i=1}^{\infty} \varepsilon_{t+i}^{r},
$$

de tal forma que el valor del coeficiente de largo plazo $\beta^{r}$ está definido por:

$$
\beta^{r}=\frac{b^{r}}{1-\rho b^{d p}} .
$$


De manera análoga, el coeficiente de largo plazo con respecto a la tasa de crecimiento de dividendos es:

$$
\beta^{d g}=\frac{b^{d g}}{1-\rho b^{d p}} .
$$

Usando datos anuales tenemos que $\rho=0,9608$, consistente con un ratio dividendo-precio de 4,08 \% anual. La estimación del vector autoregresivo para datos anuales arroja que:

$$
\Lambda=\left[\begin{array}{ccc}
0 & 0 & 0,3615 \\
0 & 0 & -0,3327 \\
0 & 0 & 0,3080
\end{array}\right],
$$

lo que implica unos coeficientes de largo plazo iguales a:

$$
\left[\begin{array}{c}
\beta^{r} \\
\beta^{d g}
\end{array}\right]=\left[\begin{array}{c}
0,5133 \\
-0,4725
\end{array}\right],
$$

que cumplen con la restricción: $\beta^{r}-\beta^{d g} \approx 1$. Este ejercicio sugiere que la volatilidad del ratio anual de dividendo-precio se explicar en partes iguales por los rendimientos y la tasa de crecimiento de dividendos futuros. La limitación de este ejercicio radica en los pocos datos anuales disponibles y, por lo tanto, en el bajo poder estadístico del proceso autoregresivo. Con el fin de subsanar este problema, la siguiente sección presenta estimadores de predictibilidad para diferentes horizontes, usando datos mensuales.

\section{B. Retornos anuales con frecuencia mensual}

Con el fin de estimar las regresiones (7), (8) y (9), se construyen alternativamente las variables de la tasa de crecimiento de dividendos y los retornos del mercado en el largo plazo como la suma de cada una de estas variables para diferentes horizontes de tiempo:

$$
\triangle d_{t}^{L P}=\sum_{j=1}^{k=24,36,48,60} \Delta d_{t+j},
$$


López-Gaviria: Predictibilidad del mercado accionario colombiano

$$
r_{t}^{L P}=\sum_{j=1}^{k=24,36,48,60} r_{t+j},
$$

donde $k$ representa un horizonte de $2,3,4$ y 5 años. ${ }^{6}$

La Tabla 2 muestra el resultado de estas regresiones para los datos del mercado accionario colombiano. Estos resultados son similares a los de Cochrane (2007) para el mercado de acciones de Estados Unidos. El rendimiento por dividendo precio pronostica retornos acumulados futuros, en vez de cambios en los dividendos futuros. El poder de predicción del rendimiento por dividendo es creciente en el horizonte de tiempo; por tanto, es informativo sobre los ciclos de retornos del mercado accionario en el largo plazo.

Tabla 2. Predictibilidad en el largo plazo

\begin{tabular}{ccc}
$r_{t \rightarrow t+k}^{m}=a+b^{r}\left(d_{t}-p_{t}\right)+\varepsilon_{t+k}$ & \\
$(\triangle d)_{t \rightarrow t+k}=a+b^{d g}\left(d_{t}-p_{t}\right)+\varepsilon_{t+k}$ & \\
$d_{t+k}-p_{t+k}=a+b^{d p}\left(d_{t}-p_{t}\right)+\varepsilon_{t+k}$ & \\
\hline Coeficientes & \\
\hline$b^{r}$ & $b^{d g}$ & \\
\hline$r_{t \rightarrow t+k}^{m}=a+b^{r}\left(d_{t}-p_{t}\right)+\varepsilon_{t+k}$ & \\
$(\triangle d)_{t \rightarrow t+k}=a+b^{d g}\left(d_{t}-p_{t}\right)+\varepsilon_{t+k}$ & \\
$d_{t+k}-p_{t+k}=a+b^{d p}\left(d_{t}-p_{t}\right)+\varepsilon_{t+k}$ & \\
0,40 & 0,11 & $(1,07)$ \\
$(4,18)$ & $(1,21)$ & $\{0,44\}$ \\
$\{1,60\}$ & {$[0,01]$} & {$[0,01]$} \\
\hline años & & Continúa
\end{tabular}

6 Dado que el coeficiente $\rho$ es cercano a 1 para series mensuales, tomar los valores de dividendos y retornos futuros sin descuento no tiene un efecto sobre los coeficientes. 
Tabla 2. Continuación

\begin{tabular}{|c|c|c|c|}
\hline \multirow[b]{2}{*}{ Horizonte $k$} & \multicolumn{3}{|c|}{ Coeficientes } \\
\hline & $b^{r}$ & $b^{d g}$ & $b^{d p}$ \\
\hline \multirow{4}{*}{3 años } & 0,52 & 0,01 & $-0,09$ \\
\hline & $(6,29)$ & $(1,64)$ & $(-1,56)$ \\
\hline & $\{1,32\}$ & $\{0,44\}$ & $\{-0,39\}$ \\
\hline & {$[0,148]$} & {$[0,01]$} & {$[0,01]$} \\
\hline \multirow{4}{*}{4 años } & 0,73 & 0,07 & $-0,14$ \\
\hline & $(6,59)$ & $(2,60)$ & $(-2,57)$ \\
\hline & $\{2,38\}$ & $\{1,33\}$ & $\{-0,66\}$ \\
\hline & {$[0,167]$} & {$[0,03]$} & {$[0,03]$} \\
\hline \multirow{4}{*}{5 años } & 0,99 & 0,07 & $-0,18$ \\
\hline & $(8,27)$ & $(2,50$ & $(-3,35)$ \\
\hline & $\{2,93\}$ & $\{1,34\}$ & $\{-1,14\}$ \\
\hline & {$[0,25]$} & {$[0,03]$} & {$[0,05]$} \\
\hline
\end{tabular}

Nota: $r_{t \rightarrow t+k}^{m}$ es el logaritmo del retorno mensual acumulado entre cada período y un horizonte $k$, descontado del promedio. $d_{t}-p_{t}$ es el logaritmo del rendimiento por dividendo descontando del promedio. $(\triangle d)_{t \rightarrow t+k}$ es la tasa de crecimiento de dividendos acumulado entre cada período y el horizonte $k$. Todos los datos son mensuales para el período abril 1995 - julio 2017. El estadístico $t$ es reportado entre paréntesis. Entre llaves está reportado el t-estadístico bajo errores estándar corregidos por autocorrelación y heterocedasticidad (Newey \& West, 1987). El $R^{2}$ de la regresión aparece entre corchetes.

Fuente: elaboración propia.

Como lo explica Cochrane (2011), el hecho que el rendimiento por dividendo pronostique retornos futuros implica que la tasa de descuento de los inversionistas es cambiante en el tiempo. Por tanto, cambios en el rendimiento por dividendo indican cambios en la prima de riesgo, la cual puede verse afectada por el ciclo económico o por la percepción de los inversionistas sobre el riesgo agregado de la economía. 


\section{Variables macroeconómicas y prima de riesgo}

Esta sección investiga cuales variables económicas contienen información sobre la prima de riesgo esperada en un horizonte futuro, más allá de la información contenida en el ratio dividendo-precio. Con este fin se estima la siguiente regresión:

$$
r_{t \rightarrow t+k}^{m}=a+\beta\left(d_{t}-p_{t}\right)+\gamma^{\prime} X_{t}+\varepsilon_{t+k},
$$

donde $X_{t}$ es una matriz que incluye variables adicionales y $\gamma$ es un vector con los coeficientes de dichas variables ${ }^{7}$ La especificación (23) es la misma usada en otros trabajos que estudian la relación de variables macroeconómicas con la prima de riesgo, como el trabajo de Rapach, Wohar y Rangvid (2005), quienes usan una muestra de 12 países desarrollados.

Del conjunto posible de variables de control, se utiliza el aporte marginal de cada una ellas al coeficiente de determinación, con el objetivo de identificar las más relevantes para el pronóstico de retornos. Esta metodología es similar a la del trabajo de Ludvigson y $\mathrm{Ng}$ (2009). ${ }^{8}$ La Figura 6 muestra las gráficas del $R^{2}$ que aporta cada variable, así como los retornos observados y pronosticados con base en las variables de control a diferentes horizontes: 2 , 3 y 5 años.

$\overline{7}$ Los resultados de este ejercicio son similares si se considera los excesos de retorno en vez de los retornos del mercado accionario.

8 Una diferencia importante con el trabajo de Ludvigson y $\mathrm{Ng}$ (2009) es que, en su trabajo, ellos usan componentes principales para agrupar las 132 variables económicas. Un ejercicio similar se realizó con las 42 series disponibles, agrupándolas en 3 componentes principales, los cuales resultan tener un alto poder explicativo de los retornos futuros del mercado accionario. Sin embargo, a diferencia del trabajo de Ludvigson y $\mathrm{Ng}$ (2009), donde cada componente tiene una interpretación económica puntual, se encontró que los componentes no tienen ninguna interpretación estructural, lo que hace que un ejercicio de esta naturaleza no pueda interpretarse. Por esta razón, se favoreció la discusión de cada una de las variables económicas por separado. 
Figura 6. Predictibilidad de mediano y largo plazo con variables macroeconómicas Panel A. Retornos acumulados a 2 años
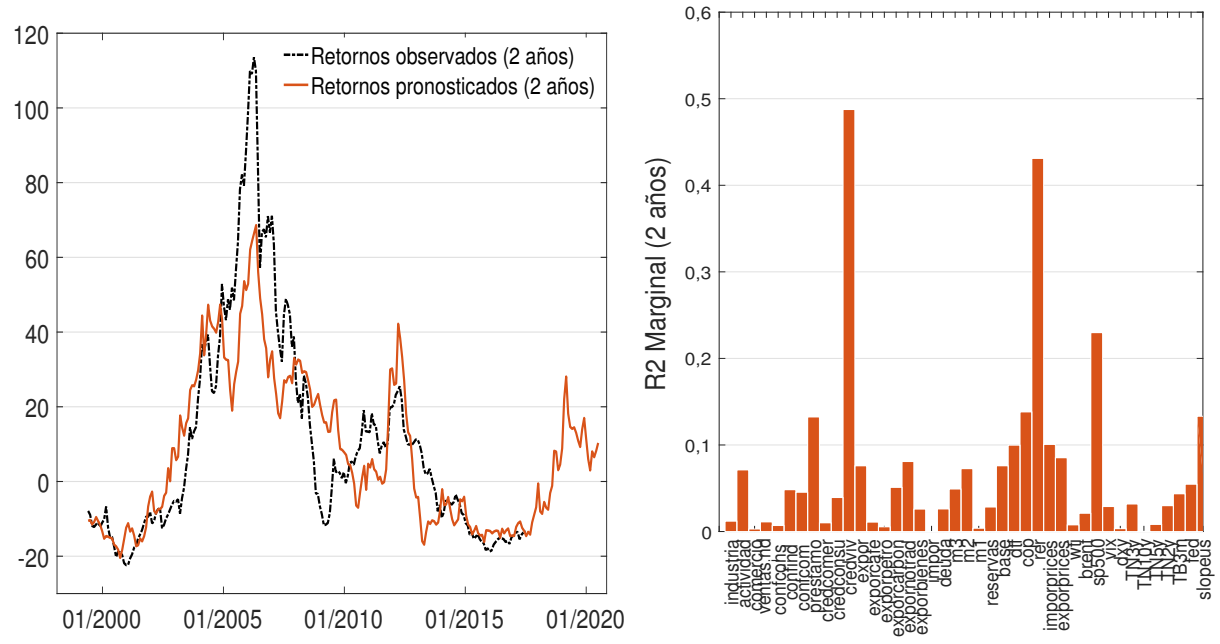

Panel B. Retornos acumulados a 3 años
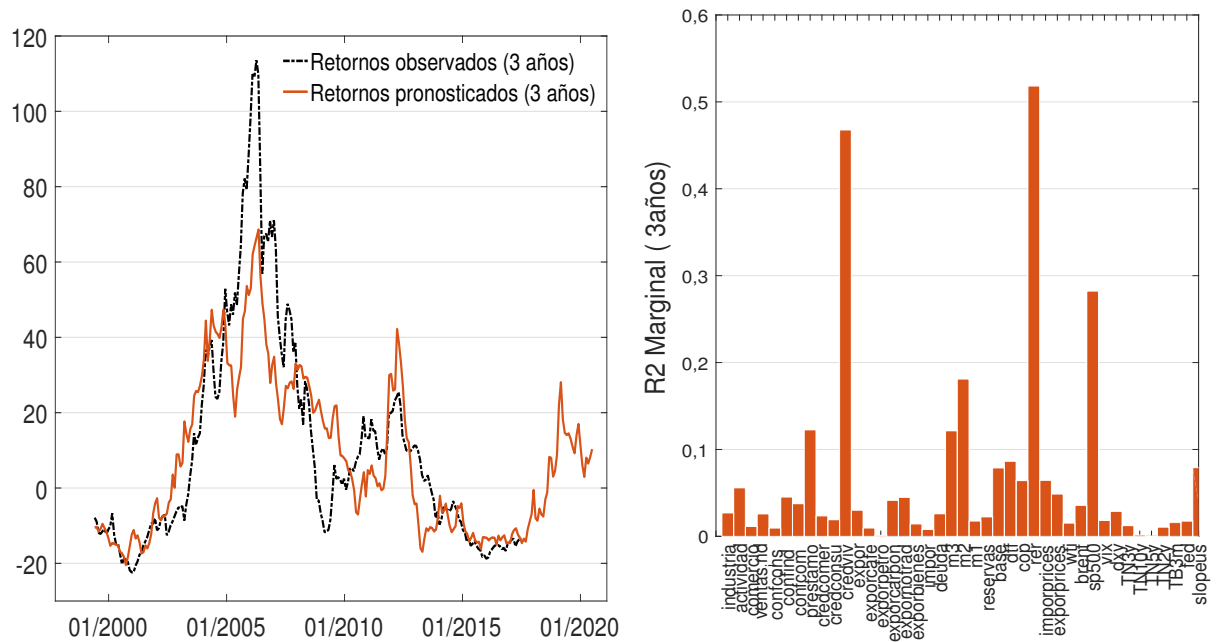

Continúa 
Figura 6. Continuación

Panel C. Retornos acumulados a 5 años
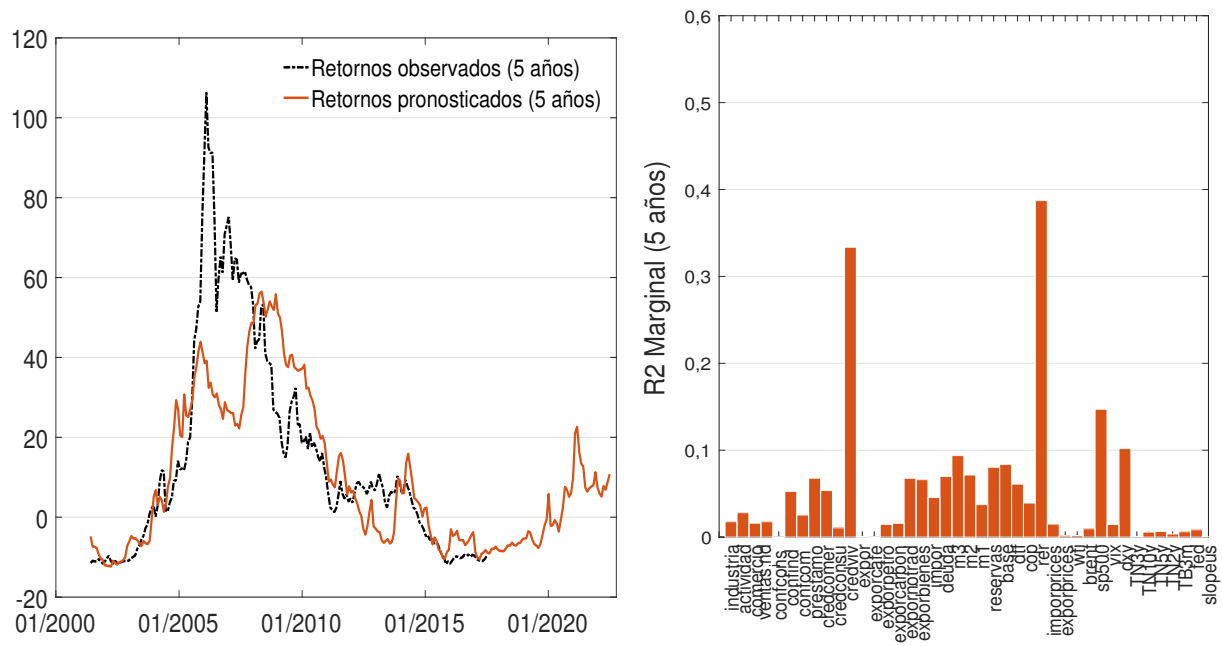

Fuente: elaboración propia.

Por su parte, la Tabla 3 presenta los resultados para cada una de las regresiones a diferentes horizontes. El rendimiento por dividendo, tiene un signo negativo y es menos significativo en los horizontes de pronóstico menores, y se vuelve positivo y altamente significativo en el horizonte a 5 años. Hay tres variables que, consistentemente, aparecen como las de mayor contribución al poder de predicción de la regresión base: la tasa de crecimiento de los créditos de vivienda, la tasa de cambio real y los retornos anuales del índice S\&P500. La tasa de crecimiento de crédito de vivienda tiene un signo negativo y es estadísticamente significativa. Este resultado sugiere que los retornos del mercado accionario están asociados a ciclos en el endeudamiento de los hogares. Una reducción en la tasa a la que crecen los préstamos hipotecarios puede sugerir un aumento en el riesgo agregado de la economía, ya sea por una desaceleración económica o por un deterioro de los balances de los hogares o del sistema financiero. 
Tabla 3. Predictibilidad de largo plazo con variables macroeconómicas

\begin{tabular}{|c|c|c|}
\hline \multicolumn{3}{|c|}{$r_{t \rightarrow t+k}^{m}=a+\beta\left(d_{t}-p_{t}\right)+\gamma^{\prime} X_{t}+\varepsilon_{t+k}$} \\
\hline \multicolumn{3}{|c|}{ Horizonte 2 años } \\
\hline Variables & Coeficientes & $\mathrm{t}$ - estadístico \\
\hline Constante $(\alpha)$ & $1,282^{* * *}$ & 6,259 \\
\hline Ratio dividendo-precio $(d-p)$ & $-0,115^{* * *}$ & $-2,561$ \\
\hline Crecimiento crédito vivienda $(C V)$ & $-0,011^{* * *}$ & $-7,431$ \\
\hline Tasa de cambio real $(R E R)$ & $-0,012^{* * *}$ & $-5,077$ \\
\hline Retorno Índice S\&P500 (SP500) & $-0,006^{* * *}$ & $-5,726$ \\
\hline$R^{2}$ & 0,647 & \\
\hline$R_{a d j}^{2}$ & 0,641 & \\
\hline \multicolumn{3}{|c|}{ Horizonte 3 ańos } \\
\hline Variables & Coeficientes $(\beta)$ & $\mathrm{t}$ - estadístico \\
\hline Constante $(\alpha)$ & $2,422^{* * *}$ & 9,709 \\
\hline Ratio dividendo-precio $(d-p)$ & $-0,086^{*}$ & $-1,694$ \\
\hline Crecimiento crédito vivienda $(C V)$ & $-0,009^{* * *}$ & $-4,920$ \\
\hline Tasa de cambio real $(R E R)$ & $-0,025^{* * *}$ & $-8,298$ \\
\hline Retorno Índice S\&P500 (SP500) & $-0,010^{* * *}$ & $-8,434$ \\
\hline$R^{2}$ & 0,756 & \\
\hline$R_{a d j}^{2}$ & 0,752 & \\
\hline \multicolumn{3}{|c|}{ Horizonte 5 años } \\
\hline Variables & Coeficientes $(\beta)$ & $\mathrm{t}$ - estadístico \\
\hline Constante $(\alpha)$ & $3,239^{* * *}$ & 10,626 \\
\hline Ratio dividendo-precio $(d-p)$ & $0,262^{* * *}$ & 4,125 \\
\hline Crecimiento crédito vivienda $(C V)$ & $-0,013^{* * *}$ & $-5,930$ \\
\hline Tasa de cambio real $(R E R)$ & $-0,033^{* * *}$ & $-9,138$ \\
\hline$R^{2}$ & 0,787 & \\
\hline$R_{a d j}^{2}$ & 0,784 & \\
\hline
\end{tabular}

Nota: $r_{t \rightarrow t+k}^{m}$ es el logaritmo del retorno mensual acumulado entre cada período y un horizonte $k$ descontado del promedio. $d_{t}-p_{t}$ es el logaritmo del rendimiento por dividendo descontando del promedio. Los datos corresponden a observaciones mensuales para el período abril 1995-julio 2017. La significancia estadística de los coeficientes está indicada por ${ }^{*}$, donde $\mathrm{p}<0,001^{* * *}, \mathrm{p}<0,05^{* *}$ y $\mathrm{p}<0,1^{*}$.

Fuente: elaboración propia. 
Una interpretación similar puede hacerse para explicar los signos negativos de las otras dos variables relevantes. Una devaluación del tipo de cambio real (una disminución del índice del tipo de cambio real) puede estar asociada con mayores riesgos, como una caída en los precios de los acciones, y, por tanto, con un aumento de los retornos futuros. Puede hacerse una interpretación similar para el caso de los retornos del mercado accionario de Estados Unidos, pues una caída en los retornos del S\&P500 puede estar asociada con un aumento del riesgo en los mercados locales, lo que conduciría a menores precios en las acciones y, por ende, a un aumento del retorno esperado. El resultado, de que los retornos del S\&P500 tienen poder predictivo en el mercado accionario local, está en línea con los resultados de Rapach et al. (2013), quienes muestran que retornos en Estados Unidos tienen un poder de predicción en otras economías (11 países industrializados). La explicación que estos autores le dan a dicho resultado tiene que ver con un proceso lento de difusión de información sobre las condiciones macroeconómicas de Estados Unidos, las cuales afectan a diferentes países vía comercial internacional.

Una de las contribuciones de este trabajo es mostrar la relación entre estas variables, que son más específicas y puntuales que las utilizadas en buena parte de la literatura, pero que al mismo tiempo ilustran la naturaleza de la prima de riesgo en una economía abierta, pequeña y emergente, como lo es la economía colombiana.

\section{Conclusiones}

Este trabajo analiza la predictibilidad del mercado accionario colombiano para un horizonte de mediano y largo plazo; con este fin, presenta un índice de precios y retornos de acciones desde 1995 hasta 2017, que incluye el ratio dividendo-precio del mercado accionado. Las regresiones de pronóstico con base en dichos retornos muestran que el poder de pronóstico aumenta en el horizonte de tiempo. Esto sugiere la existencia de ciclos de mediano y largo plazo en los retornos, lo que puede explicarse por variaciones en el tiempo en la prima de riesgo. Dichas variaciones son consistentes con el hecho de que los movimientos en el ratio dividendo-precio del mercado son explicados, 
en su mayoría, por cambios en los retornos futuros. Adicionalmente, este trabajo encuentra que, a la hora de pronosticar retornos futuros, de una muestra grande de indicadores económicos, son las variables relacionadas con el crédito hipotecario, la tasa de cambio real y los retornos del mercado accionario de los Estados Unidos las que contienen información adicional a la presente en el ratio dividendo-precio.

Esto sugiere que modelos de la prima de riesgo de los inversionistas del mercado accionario colombiano deben incorporar elementos relacionados al mercado de crédito y tener en cuenta el hecho de que Colombia es una economía pequeña y abierta. Se concluye con este trabajo que el mercado accionario tiene ciclos de largo plazo que están fuertemente influenciados por el crédito financiero y la primad de riesgo internacional.

Una discusión sobre la estabilidad de los pronósticos y el ajuste de los mismos para periodos fuera de la muestra están más allá del alcance de este documento, pero son temas interesantes para futuros trabajos de investigación. Finalmente, el análisis sobre el desempeño, no del mercado agregado, sino de los emisores en particular, puede ser un complemento a los resultados de este trabajo.

\section{Anexo}

Tabla 1A. Listado de las variables económicas

\begin{tabular}{ll}
\hline Variables & \\
\hline Producción industrial & Reservas Internacionales \\
Índice de actividad económica & Base Monetaria \\
Ventas del comercio & Tasa DTF \\
Confianza consumidores & Tipo de Cambio (COP/USD) \\
Confianza industriales & Tasa de Cambio Real \\
Confianza comerciantes & Precio Importados \\
Prestamos no rentables & Precio Exportados \\
\hline
\end{tabular}

Continua 
López-Gaviria: Predictibilidad del mercado accionario colombiano

Tabla 1A. Continuación

\begin{tabular}{ll}
\hline Variables & \\
\hline Crédito de comercio & Precio Petróleo WTI \\
Crédito de consumo & Precio Petróleo Brent \\
Crédito de vivienda & Retorno Índice S\&P500 \\
Exportaciones totales & Índice volatilidad VIX \\
Exportaciones café & Índice precio del dólar DXY \\
Exportaciones petróleo & Tasa de los tesoros americanos a 3 meses \\
Exportaciones carbón & Tasa de los tesoros americanos a 2 años \\
Exportaciones no tradicionales & Tasa de los tesoros americanos a 3 años \\
Exportaciones bienes fabricados & Tasa de los tesoros americanos a 5 años \\
Importaciones & Tasa de los tesoros americanos a 10 años \\
Deuda externa & Tasa de los Federal Funds \\
M1 & Pendiente de la curva tesoros americanos \\
M2 & Pendiente de la curva local de TES \\
M3 & \\
\hline
\end{tabular}

Nota: todas las variables son de frecuencia mensual para el período mayo 1996-julio 2017. Las variables no estacionarias, como los saldos de crédito, los niveles de exportaciones o importaciones, el saldo de deuda y los agregados monetarios, entre otros, son transformadas a estacionarias tomando la tasa de crecimiento anual.

Fuente: elaboración propia con datos tomados de Bloomberg.

\section{Referencias}

Alonso, J. C. \& García, J. (2009). ¿Qué tan buenos son los patrones del IGBC para predecir su comportamiento?: Una aplicación con datos de alta frecuencia. Estudios Gerenciales, 25(11),13-36.

Ang, A. \& Bekaert, G. (2007). Stock return predictability: Is it there? The Review of Financial Studies, 20(3), 651-707. 
Arango, L. E., González, A. \& Posada, C. (2002). Returns and the interest rate: a non-linear relationship in the Bogotá stock market. Applied Financial Economics, 12(11), 835-842.

Bastidas, A. (2008). Incertidumbre de la prima de riesgo del mercado accionario de Colombia 1991-2007. Perfil de Coyuntura Económica, 12, 159-178.

Boyd, J., Hu, J. \& Jagannathan, R. (2005). The Stock Market's Reaction to Unemployment News: Why Bad News Is Usually Good for Stocks. The Journal of Finance, 60(2), 649-672.

Campbell, J. Y. \& Ammer, J. (1993). What Moves the Stock and Bond Markets? A Variance Decomposition for Long-Term Asset Returns. The Journal of Finance, 48(1), 3-37.

Campbell, J. Y. (1987). Stock Returns and the Term Structure. Journal of Financial Economics, 18(2), 373-399.

Campbell, J. Y. (1990). Measuring the Persistence of Expected Returns (NBER working paper No. 3305). Recuperado del sitio web The National Bureau of Economic Research: https://www.nber.org/papers/w3305

Campbell, J. \& Shiller, R. (1988a). The Dividend-Price Ratio and Expectations of Future Dividends and Discount Factors. The Review of Financial Studies, 1(3), 195-228.

Campbell, J. \& Shiller, R. (1988b). Stock Prices, Earnings, and Expected Dividends. The Journal of Finance, 43(3), 661-676.

Chen, N.-F., Roll, R. \& Ross, S.A. (1986). Economic Forces and the Stock Market. Journal of Business, 59(3), 383-403.

Cochrane, J. (2007). The Dog that Did Not Bark: A Defense of Return Predictability. The Review of Financial Studies, 21(4), 1533-1575.

Cochrane, J. (2011). Presidential Address: Discount Rates. The Journal of Finance, 66(4), 1047-1108. 
Cutler, D., Poterba, J. \& Summers, L. H. (1989). What Moves Stock Prices? Journal of Portfolio Management, 15(2), 4-12.

Fama, E. F. (1981). Stock Returns, Real Activity, Inflation, and Money. The American Economic Review, 71(4), 545-565.

Fama, E. F. \& Schwert, G. (1977). Asset returns and inflation. Journal of Financial Economics, 5(2), 115-146.

Geske, R. \& Roll, R. (1983). The Fiscal and Monetary Linkage between Stock Returns and Inflation. The Journal of Finance, 38(1), 1-33.

Gómez-Sánchez, A.M. \& Astaiza-Gómez, J. (2015). Primas de riesgo de renta variable ex-post y ciclos económicos en Colombia: Una investigación empírica utilizando los filtros de Kalman y Hodrick-Prescott. Revista Finanzas y Politica Económica, 7(1), 109-129.

Goyal, A. \& Welch, I. (2003). Predicting the Equity Premium with Dividend Ratios. Management Science, 49(5), 639-654.

Harvey, C. (1991). The World Price of Covariance Risk. The Journal of Finance, 46(1), 111-157.

Hjalmarsson, E. (2010). Predicting Global Stock Returns. Journal of Financial and Quantitative Analysis, 45(1), 49-80.

Kristjanpoller, W. \& Muñoz, R. (2012). Analysis of Day of the Week Effect in the main Latin-American stock markets: an approximation through the Stochastic Dominance Criterion. Estudios de Economia, 39(1), 5-26.

Larrain, B. \& Yogo, M. (2008). Does firm value move too much to be justified by subsequent changes in cash flow? Journal of Financial Economics, 87(1), 200-226.

Lettau, M. \& Ludvigson, S. (2005). Expected returns and expected dividend growth. Journal of Financial Economics, 76(3), 583-626.

Lo, A. W. \& MacKinlay, A. (1988). Stock Market Prices do not Follow Random Walks: Evidence from a Simple Specification Test. The Review of Financial Studies, 1(1), 41-66. 
Ludvigson, S. C. \& Ng, S. (2009). Macro Factors in Bond Risk Premia. The Review of Financial Studies, 22(12), 5027-5067.

Montenegro, A. (2007). El efecto día en la bolsa de valores de Colombia (Documentos de Economía No. 2007-09). Recuperado del Departamento de Economía, Pontificia Universidad Javeriana, Bogota.

Nelson, C. R. (1976). Inflation and Rates of Return on Common Stocks. The Journal of Finance, 31(2), 471-483.

Newey, W. \& West, K. (1987). A Simple, Positive Semi-Definite, Heteroskedasticity and Autocorrelation Consistent Covariance Matrix. Econometrica, 55(3), 703-708.

Ochoa, C. M. \& Avendaño, G. I. (2005). The Unification of the Colombian Stock Market: A Step Towards Efficiency-Empirical Evidence. Latin American Business Review, 5(4), 69-98.

Ospina, J. (2007). Características generales del mercado accionario colombiano como mercado emergente. Economía y Desarrollo, 6(1), 105-136.

Pearce, D. \& Roley, V. (1983). The Reaction of Stock Prices to Unanticipated Changes in Money: A note. The Journal of Finance, 38(4), 1323-1333.

Perez-Villalobos, J. \& Mendoza-Gutiérrez, J. (2010). Efecto día en el mercado accionario colombiano: una aproximación no paramétrica (Borradores de Economía, No. 585). Recuperado del Banco de la República: http://www.banrep.gov.co/es/borrador-585

Rangvid, J., Schmeling, M. \& Schrimpf, A. (2014). Dividend Predictability Around the World. Journal of Financial \& Quantitative Analysis, 49(5-6), 1255-1277.

Rapach, D., Strauss, J. \& Zhou, G. (2013). International Stock Return Predictability: What Is the Role of the United States? The Journal of Finance, 68(4), 1633-1662. 
Rapach, D., Wohar, M. \& Rangvid, J. (2005). Macro variables and international stock return predictability. International Journal of Forecasting, 21(1), 137-166.

Restrepo, M., Zuluaga, S. \& Guerra, M. (2002). El mercado de capitales colombiano en los noventa y las firmas comisionistas de bolsa. Colección Economía Colombiana. Bogotá. Fedesarrollo.

Sierra, K., Duarte, J. \& Ortiz, V. (2015). Predictibilidad de los retornos en el mercado de Colombia e hipótesis de mercado adaptativo. Estudios Gerenciales, 31(137), 411-418.

Solnik, B. (1993). The performance of international asset allocation strategies using conditioning information. Journal of Empirical Finance, 1(1), $33-55$.

Vélez-Pareja, I. (2000). The Colombian Stock Market: 1930-1998. Latin American Business Review, 1(4), 61-84.

Yepes-Rios, B., Gonzalez-Tapia, K. \& Gonzalez-Perez, M. (2015). The integration of stock exchanges: The case of the Latin American Integrated Market (MILA) and its impact on ownership and internationalization status in Colombian brokerage firms. Journal of Economics, Finance and Administrative Science, 20(39), 84-93. 